\title{
Aktualität des Naturrechts?
}

Hans-Dieter Mutschler

Die meisten Philosophen und Theologen schalten ab, wenn vom "Naturrecht" die Rede ist. Sie unterstellen, dass es sich dabei um eine konservativ-katholische, mittelalterliche Doktrin handle, die man heute sinnvollerweise nicht mehr vertreten könne. Wir werden sehen, dass dieser Vorwurf gegen manche Vertreter des Naturrechts berechtigt ist, nicht aber gegen das, was man den "harten Kern« des Naturrechts nennen könnte.

Dieser besteht in der seit der Antike diskutierten Frage, ob all unsere moralischen und juridischen Normen nur willkürlich vom Menschen gesetzt seien, wie die Sophisten annahmen ("thesei«) oder ob es auch Normen gebe, die unabhängig davon gelten ("physei«), wie Plato unterstellte. Letztere Position vertrat auch Aristoteles in seiner Nikomachischen Ethik. ${ }^{1}$ Letzteres liegt der Vorstellung von einer nicht verlierbaren Menschenwürde und einem nicht verlierbaren Menschenrecht zugrunde.Von daher ist nicht leicht zu sehen, wie man an ihnen festhalten sollte, um dennoch das Naturrecht abzulehnen. Die klassische Formulierung des Naturrechts stammt von den Stoikern und lautet "secundum naturam vivere", wobei nicht so recht klar ist, was hier unter "Natur" zu verstehen sei.

Zuvor aber vielleicht eine Skizze zweier gegensätzlicher Extrempositionen, die beide zu vermeiden sind und die die Diskussion in die falsche Richtung treiben. Wir könnten sie die "konservativkatholische" und die "radikal-protestantische" Richtung nennen.

Die konservativ-katholische Richtung orientiert sich an Thomas von Aquin. Die Naturrechtslehre bei Thomas hat ganz verschiedene Aspekte, aber derjenige, der uns zunächst einmal beschäftigen sollte, ist der kosmologische, der auf Aristoteles zurückgeht. Danach hat alles im Universum seinen Sinn und Zweck und seine ungeschichtliche Essenz, die ein geordnetes Reich der substanziellen Formen bildet. Es ist nach dieser Auffassung so, dass alles ein Gut hat, selbst die Steine. Diese streben nach ihrem "natürlichen Ort«, nämlich möglichst tief zu fallen. Das menschliche Handeln rangiert sich ein in diesen allgemeinen Trend hin zum Guten, das sich beim Menschen zur Moralität steigert. Wir haben also hier ein Konzept, dem

1 Er rechnet allerdings auch die Sklaverei und die Versklavung von Kriegsgefangenen zum Naturrecht.Vgl. Erik Wolf/Reinhard Brandt u.a., Art. Naturrecht, in: HPW 6 (1984), 560-623, hier Brandt, II. Antike, 566. 
die moderne Unterscheidung zwischen Sein und Sollen völlig fremd ist. Das Sein ist nach Thomas - omne ens bonum - von sich her wertdurchdrungen, so dass der Mensch als moralisch Handelnder nicht aus der kosmologischen Ordnung herausfällt. In einer solchen Konzeption ist das, was wir heute "Naturrecht" nennen, nicht nur eine Eigenschaft des von der Natur isolierten Menschen, sondern eine Eigenschaft der äusseren Natur als Ganzer, die im Sinn einer "natürlichen Theologie« auf Gott verweist. ${ }^{2}$

Wir sollten heute unterscheiden zwischen den "Dingen der $\mathrm{Na}-$ tur" und der "Natur der Dinge«. Die "Dinge der Natur", das sind Elementarteilchen, Wolken, Spatzen und Vulkane, die "Natur der Dinge«, das sind ihre Wesenseigentümlichkeiten, also das, was sie zu dem macht, was sie sind. Das Irritierende bei Thomas und seinen Nachfolgern ist, dass sie diese Unterscheidung nicht kennen oder ignorieren. Bei Thomas versteht sich das von selbst. Er kannte noch keine autonome Wissenschaft der Natur, die sich mit den Phänomenen beschäftigt, um ihr inneres Wesen in produktiver Ignoranz auf sich beruhen zu lassen. Für ihn war die Doktrin vom Wesen der Dinge zugleich Wissenschaft von der Natur. Wer allerdings noch heute eine so geartete Naturrechtslehre vertritt, muss sich den Vorwurf gefallen lassen, dass er die eigenständige Dynamik der modernen Naturwissenschaft nicht zur Kenntnis genommen hat.

Der prominenteste Vertreter einer konservativ-katholischen, an Thomas orientierten, Naturrechtslehre ist der Philosoph Robert Spaemann. ${ }^{3}$ Von Thomas unterscheidet ihn, dass er die Teleologie aus dem anorganischen Bereich herausnimmt, um sie auf das Lebendige zu beschränken. Aber auch so gesehen wird das Naturrecht in

\footnotetext{
2 Ein solcher Blick aufThomas ist freilich strittig. Nach Kluxen hat erst der Neothomismus es so dargestellt, während Thomas die Ethik von der Metaphysik abgekoppelt habe. Wolfgang Kluxen, Moral, Vernunft, Natur, Paderborn 1997. Sei dem, wie ihm wolle, ob original-thomasisch oder neothomistisch, die genannte Position gibt es bis heute im von mir "konservativ" genannten Katholizismus.

3 Eine kurze Zusammenfassung dazu bietet Robert Spaemann, Die Aktualität des Naturrechts, in: Naturrecht in der Kritik, hg. v. Ernst-Wolfgang Böckenförde/Franz Böckle, Mainz 1973, 262-276; Warum gibt es kein Recht ohne Naturrecht?, in: Naturrecht und Kirche im säkularen Staat, hg. v. Hans-Gregor Nissing, Wiesbaden 2016, 27-34. Ausführlich stellt Spaemanns Naturrechtslehre dar: Andrzej Kucinsky, Naturrecht in der Gegenwart. Anstösse zur Erneuerung naturrechtlichen Denkens im Anschluss an Robert Spaemann, Paderborn 2017.
} 
der äusseren Natur verwurzelt. ${ }^{4}$ Er fordert deshalb die "Erneuerung eines teleologischen Naturbegriffs $\aleph^{5}$.

Die Problematik, die hierin liegt, wurde deutlich beim Besuch des letzten Papstes, Benedikt XVI, im Bundestag Berlin im September 2011. Spaemann gilt allgemein als dessen Ghostwriter und so vertrat der Papst vor dem Bundestag eine Position, die den Zuhörern unverständlich bleiben musste. Es war übrigens die Partei der Grünen, die vor allem gegen die Anwesenheit des Papstes im Bundestag protestierten und so schien es ein geschickter Schachzug, dass Benedikt zunächst einmal die Grünen für ihr ökologisches Engagement lobte. Doch dann fuhr er fort, man müsse von einer "Ökologie der Natur» zu einer "Ökologie des Menschen" übergehen. Was könnte damit gemeint sein?

Dieser Übergang setzt den thomistischen Naturbegriff der Essenzen und der durchgängigen Teleologie voraus. Das Gut des Lebendigen weist voraus auf das moralisch zu verstehende Gut des Menschen im Sinn einer Naturrechtslehre. Es wird kaum jemand im Saal gegeben haben, der das wirklich verstanden hat, insbesondere nicht die Grünen, denn diese Idee einer durchgängigen Teleologie des Lebendigen ist in unserer Kultur aus guten Gründen nicht mehr präsent und wie sich zeigen wird, hängt das richtig verstandene $\mathrm{Na}$ turrecht auch nicht an einer solchen Vorstellung.

Sie ist zudem gefährlich. Macht man nämlich keine Differenz zwischen der Natur der Dinge und den Dingen der Natur, dann läuft man ständig die Gefahr naturalistischer Fehlschlüsse. Dann darf der Mensch nur Geschlechtsverkehr zum Zwecke der Zeugung des Nachwuchses haben, weil das bei den Tieren auch so ist oder man muss empfängnisverhütende Massnahmen verbieten, weil sie in der Natur nicht vorkommen. Aber dann sollte der Papst auch kein Flugzeug besteigen. ${ }^{6}$

Dass das Naturrecht nicht an einer Gesamtteleologie der Natur hängt, sieht man bei Kant. Seine Bestimmungen von Achtung und Würde, die dem Menschen an sich zukommen, hängen nicht an einer solchen Gesamtteleologie, die es bei ihm nicht gibt, weshalb er auch

\footnotetext{
4 Auf ganz andere Art findet man diesen Gedankengang bei Hans Jonas, Organismus und Freiheit. Ansätze zu einer philosophischen Biologie, Göttingen 1973. Der Versuch einer Aktualisierung findet sich in: Naturphilosophie als Grundlage der Naturethik. Zur Aktualität von Hans Jonas, hg. v. Gerald Hartung u.a.,Freiburg 2013, allerdings ohne auf die Schwierigkeiten des Naturrechtsbegriffs einzugehen.

5 Spaemann, Aktualität des Naturrechts, 268.

${ }^{6}$ In der Enzyklika humanum vitae Papst Pauls VI von 1968 wird das Verbot des Geschlechtsverkehrs aus rein hedonistischen Gründen mit der biologischen »Natur» des Menschen begründet.
} 
ein Gegner des teleologischen Gottesbeweises war. Die Abkoppelung des Naturrechtsgedankens von einer solchen Form der natürlichen Theologie gibt es schon bei Samuel von Pufendorf im 17. Jahrhundert, vor allem aber in der Deklaration der Menschenrechte in der Französischen Revolution. In diesem Zusammenhang waren die Menschenrechte eine Brechstange gegen Adel und Klerus, d.h. eben keine konservativ-katholische Sonderlehre. Auch die Marxisten beriefen sich auf die Menschenrechte. Die letzte Strophe der »Internationale« lautet:

Völker, hört die Signale!

Auf zum letzten Gefecht!

Die Internationale

erkämpft das Menschenrecht.

Man kann sich fragen, wie die Basis-Überbau-Lehre der Marxisten mit einem ahistorisch zu verstehenden Menschenrecht verträglich ist. Aber der Fall zeigt, dass Menschenwürde und Menschenrechte kein Fall für den politisch-religiösen Konservativismus sind. Und so schrieb denn Ernst Bloch ein eigenes Werk über "Naturrecht und menschliche Würde« (1961).

Die konservativ-katholische Position ist also überschwänglich und aus den genannten Gründen abzulehnen. Hinzugefügt muss werden, dass sicher keine Mehrheit der katholischen Theologen eine solche konservative Position akzeptieren wird. ${ }^{7}$ Sie wurde erwähnt, um eine Extremposition zu kennzeichnen.

Die andere Extremposition wäre die radikal protestantische, die sich natürlich auch nicht bei allen evangelischen Theologen findet, umso mehr, als dass sowohl Luther, Melanchthon, aber auch Zwingli eine Naturrechtslehre kennen. Eine radikal protestantische Position vertritt allerdings Ingolf Dalferth in einer Monographie zum Thema. Sein entscheidendes Prinzip einer sola-fide-Konzeption lautet: "Dass die Welt Gottes Schöpfung ist, ist ihr nicht anzusehen [...] Wer sich als Geschöpf versteht, der sieht Gott überall im Spiel, und wer es nicht tut, nirgends. $\|^{8}$

Um dies durchzuhalten, bestreitet er, dass der Mensch durch feststehende Wesensmerkmale ausgezeichnet sei. Weder durch Vernunft, noch durch Freiheit oder Würde. All diese Eigenschaften seien kon-

\footnotetext{
7 Weshalb namhafte katholische Moraltheologen schon seit Langem das Naturrecht von der Kosmologie abkoppeln: Böckenförde/Böckle (Hg.), Naturrecht in der Kritik; Wilhelm Korff, Theologische Ethik, Freiburg 1975.

${ }^{8}$ Ingolf U. Dalferth, Naturrecht in protestantischer Perspektive, Baden Baden 2008, $44 f$.
} 
tingent in dem Sinn, dass sie graduell aus Vorformen im Tierreich hervorgegangen seien - es fällt das Wort "Emergenz - man könne deshalb nicht von einer "Natur des Menschen sprechen. $^{9}$

Diese prinzipielle Kontingenz des Menschen wird dann natürlich auch auf die Normen des Handelns bezogen: Keine Werte sind von Gott gesetzt, sondern der Mensch setzt die Werte selber. Das gilt auch für die Menschenwürde. Menschenwürde ist eine Setzung aus bestimmten Erfahrungen heraus. Statt von Gott gesetzten Werten gehe man im Protestantismus von einer "Fundierung des positiven Rechts in der göttlichen Gabe der Freiheit" aus. ${ }^{10}$ Man hat das auch die »evangelische Rechtstheologie» genannt.

Um die Differenz zu Thomas überdeutlich zu machen, rechnet Dalferth die 10 Gebote, nicht wie dieser, zum Naturrecht: »Der Mensch wird im Glauben nicht dem Dekalog unterstellt, wie Luther betonte, sondern er wird im Glauben dazu befreit, selbst neue Dekaloge zu schaffen." Das einzige, prinzipiell geltende Gebot des Dekalogs ist das erste. Uns wird am Ende des Buches nochmal explizit gesagt, dass die Menschenwürde ein theologisches Datum sei. ${ }^{11}$

Diese Konzeption zieht elementare Einwände auf sich. Wenn die Menschenwürde ein theologisches Datum ist, was ist dann mit Nicht- oder Andersgläubigen? Haben sie etwa keine Würde? Und wenn die Menschenwürde nur eine Konvention ist, dann kann sie auch abgeschafft werden, wie jede Konvention. Dann waren die Väter des Grundgesetzes im Irrtum, wenn sie nach den schrecklichen Erfahrungen des III. Reiches gleich im ersten Artikel des Deutschen Grundgesetzes die Würde des Menschen als »unantastbar" bezeichneten und wenn wir z.B. die Menschenrechtsverletzungen in China anprangern, dann haben die Chinesen recht, wenn sie Menschenwürde und Menschenrechte für eine Erfindung des Westens halten, an die sie nicht gebunden seien.

Dalferth verweist immer wieder darauf, dass die Menschenrechte historisch variabel seien. Das ist so. Menschenwürde und Menschenrechte verhalten sich wie Grundsatz und Anwendung. Anwendungen haben immer etwas Kontingentes. Spätestens seit der Französischen Revolution verbinden wir beides, aus der Einsicht heraus, dass ein Mensch, dem man keine minimalen Rechte zu-

9 Ebd., 11, 17, 42.

${ }^{10}$ Ebd., 44, 55, 61, 63.

${ }^{11}$ Ebd.,7f, 66. Auch die Theologin Tietz vertritt eine ähnliche Position, wälzt aber die Begründung der Menschenwürde auf die Diskursethik Habermas' ab: Christiane Tietz, Why Should Naturally Moral Laws Exist?, in: Concepts of Law in the Sciences, Legal Studies and Theology, hg. v. Michael Welker, Tübingen 2013, 355-371, hier 371. Es wird sich zeigen, dass das keine Lösung sein kann. 
spricht, das Bewusstsein seiner Würde verlieren könnte. Von daher fassen wir heute die Menschenwürde als eine Disposition, nicht als einen aktuellen Besitz, wie Lunge und Leber. ${ }^{12}$

Aber daraus folgt nicht, dass alle Menschenrechte kontingent sind. In der Deklaration der Vereinten Nationen von 1948 zählt man unter die Menschenrechte das Verbot von Folter, Sklaverei und religiöser Bevormundung. Wenn Dalferth recht hätte, dann wäre eine Gesellschaft denkbar, in der all dies zu Recht erlaubt wäre. Kann man sich das vorstellen?

Was die 10 Gebote anbelangt, so sind sie nach Thomas von Aquin der natürlichen Vernunft als Gesollte einsichtig. Er geht weiter davon aus, dass es sogenannte "inclinationes naturales" gibt ${ }^{13}$, d.h. natürliche Neigungen des Menschen, nicht belogen, betrogen, bestohlen oder gar getötet zu werden. Diese Seite von Thomas' Naturrechtslehre ist nach wie vor aktuell, wie sich noch zeigen wird. Aber der Radikalprotestant muss all dies bestreiten, obwohl doch solche "inclinationes naturales" in fast allen Ethikkodizes zu finden sind. Nur deshalb konnte Hans Küng ein "Projekt Weltethos « formulieren, das zwar viel (berechtigte) Kritik erfuhr, aber nicht in dieser Hinsicht. Zu den allgemein verbreiteten Normen gehört vor allem die "Goldene Regel«, die Kant später zum kategorischen Imperativ im Sinn einer Verallgemeinerbarkeit der Handlungsmaximen verschärfte. All dies wird der Radikalprotestant vom Tisch wischen.

Um jeden Anschein eines Essentialismus zu vermeiden, lehrt Dalferth sogar die Kontingenz der Vernunft als ein Evolutionsprodukt. Das ist einigermassen erstaunlich, wenn man an das Scheitern der Soziobiologie und an die immensen Probleme denkt, den Menschen zu naturalisieren, und das Zauberwort "Emergenz " hilft hier auch nicht weiter, denn entweder ist damit "schwache Emergenz" gemeint, dann setzt sie die durchgeführte Naturalisierung des Menschen voraus, von der keine Rede sein kann, oder man meint damit "starke Emergenz«, dann erklärt sie gar nichts. ${ }^{14}$

Summa: wir sollten die Extreme des katholischen Konservativismus und des Radikalprotestantismus meiden. Sie führen beide in unlösbare Aporien hinein. Was wäre dann eine vernünftige mittlere

\footnotetext{
${ }^{12}$ Bei Pollmann/Menke heisst es: Die Menschenwürde ist "weder Mitgift, noch Leistung noch Eigenschaft, sondern ein in Realisierung begriffenes Potential [...] Sie ist »ein zerbrechliches Gut - eben darum ist sie auf rechtliche Sicherung und soziale Schonung angewiesen." Arnd Pollmann/Christoph Menke, Philosophie der Menschenrechte, Hamburg 2007, $146 f$.

${ }^{13} \mathrm{Vgl}$. Thomas S.Th. I-II, q. 94, a $3 \mathrm{f}$.

${ }^{14}$ Ich habe das näher ausgeführt: Hans-Dieter Mutschler, Halbierte Wirklichkeit. Warum der Materialismus die Welt nicht erklärt, Darmstadt 2014.
} 
Position? Um dies zu sehen, ist es vielleicht sinnvoll, erst einmal namhafte Philosophen zu überprüfen, die das Naturrecht weit von sich weisen, wie sich aber zeigen wird, dennoch versteckt von ihm zehren.

Jürgen Habermas wurde bekannt und zumeist akzeptiert für seine These vom "nachmetaphysischen Denken ${ }^{15}$. Unter "Metaphysik" versteht er die traditionelle Auffassung, wonach im Kosmos alles sinnvoll aufeinander hingeordnet sei, so dass der Mensch in dieser Ordnung seinen gebührenden Platz finde, also das, was wir hier die »konservativ katholische Position" genannt haben. Sieht man es so, dann ist das Naturrecht überholt und in der Tat ist das Habermas' Auffassung. Es gebe "zum nachmetaphysischen Denken keine Alternative«. Das ist natürlich der Fall, wenn man "Metaphysik" so stark orchestriert wie er es tut: Es gebe drei Grundmotive des metaphysischen Denkens: "das Einheitsmotiv der Ursprungsphilosophie", "die Gleichsetzung von Sein und Denken", "die Heilsbedeutung der theoretischen Lebensführung" = "Identitätsdenken, Ideenlehre und starker Theoriebegriff " ${ }^{16}$. Dass es auch schlankere Formen von Metaphysik gibt, hat Habermas niemals zur Kenntnis genommen.

Er ersetzt weiter alle Formen substanzieller Subjektivität durch eine "prozedurale ${ }^{17}$ Vernunft. Diese ist dem Vorgehen der Naturwissenschaft nachgebildet. Der Naturwissenschaftler unterstellt von Vornherein nichts Inhaltliches im Sinn der alten Rationalisten, sondern er hält sich an bewährte Methoden, die ihm erlauben, empirisches Wissen zu generieren. Auf diese Art hat die Philosophie, insbesondere die Moralphilosophie, bei Habermas keinen apriorischen Charakter, sondern sie bedient sich nach dem "linguistic turn" des zwanglosen Diskurses, um ihre Resultate allererst hervorzubringen.

Ausgangspunkt hier ist die nicht zu leugnende Tatsache, dass wir sprachliche, auf Verständigung hin angelegte, Wesen sind. Also ist es naheliegend, alle zu begründenden Normen im zwanglosen, nicht durch Machtverhältnisse verzerrten, Diskurs zu gewinnen. So weit, so einsichtig.

Nun ist aber Habermas zugleich Kantianer. Danach müssen Moralprinzipien ausnahmslos gelten und sind nicht einfach nur empirische Verallgemeinerungen: "Die Intuition, die sich in der Idee der Verallgemeinerungsfähigkeit von Maximen ausdrückt, meint mehr: gültige Normen müssen die Anerkennung von Seiten aller

15 Jürgen Habermas, Moralbewußtsein und kommunikatives Handeln [1983a], Frankfurt ${ }^{4} 1991$.

${ }^{16}$ Ders., Nachmetaphysisches Denken. Philosophische Aufsätze, Frankfurt 1983b, 36.

17 Ders., Moralbewußtsein, 44. 
Betroffenen verdienen. «18

Dann kann sich aber die Konstitution unserer Moralprinzipien nicht dem kontingenten Faktum bloss empirischer Übereinkunft verdanken, denn daraus liesse sich keine ausnahmslose Geltung ableiten. Es ist folglich so, dass Habermas seine sprachphilosophische Konstitution des Diskursprinzips metaphysisch gemeint haben muss. Der Mensch ist also nicht nur faktisch, sondern notwendig auf Sprache, Normativität und Verständigung hin ausgerichtet. Folglich handelt es sich um naturrechtliche Bestimmungen als Wesenseigentümlichkeiten des Menschen. Diese versteckte Metaphysik verrät sich bei Habermas auf Schritt und Tritt so, wenn er von der »idealen Kommunikationsgemeinschaft" spricht usw. An manchen Stellen ist sich Habermas dieses Rückgriffs auf die Tradition bewusst: So spricht er von einem »Vernunftideal«, das einen »Rest von Metaphysik « beinhalte, ohne den man nicht auskomme. ${ }^{19}$

An dieser Stelle war sein Freund und Inspirator Karl-Otto Apel klarer. Apel lässt keinen Zweifel darüber, dass seine Letztbegründung im Rahmen einer "Transzendentalpragmatik" metaphysischen Charakter hat. ${ }^{20}$ Wenn es bei dem Habermasschüler Konrad Ott heisst, dass in der Diskursethik Menschenwürde bzw. die Menschenrechte erst im Diskurs begründet werden müssen, so übersieht er dieses versteckte Apriori, das sie bereits enthält. ${ }^{21}$

Der Philosoph Christian Illies macht zu Recht darauf aufmerksam, dass im strengen Verallgemeinerungsprinzip moralischer Normen die Menschenwürde bereits enthalten sei. ${ }^{22}$ Wenn jeder Mensch notwendigerweise und im selben Sinn Adressat derselben Normen ist, so hat er als solcher auch dieselbe Würde, wie schon Kant bemerkte. $^{23}$

\footnotetext{
${ }_{18}$ Ders., Nachmetaphysisches Denken, 77.

19 Ders., Moralbewußtsein, 184.

${ }^{20}$ In seinem Buch über Peirce sagt Apel im zustimmenden Sinn: „Der einzige Schutz vor Metaphysik ist nach Peirce letztlich Metaphysik. "Karl-Otto Apel, Der Denkweg von Charles S. Peirce. Eine Einführung in den amerikanischen Pragmatismus, Frankfurt 1975, 31. Überflüssig zu sagen, dass Peirces Metaphysik von ganz anderer Art ist als die des Thomas von Aquin.

${ }^{21}$ Vgl. Konrad Ott, Moralbegründungen, Hamburg 2001, 177. Die Differenz zu Apel wird gut herausgearbeitet bei Walter Reese-Schäfer, Karl-Otto Apel zur Einführung, Hamburg 1990, 95-102.

${ }^{22}$ Vgl. Christian Illies, Philosophische Anthropologie im biologischen Zeitalter. Zur Konvergenz von Moral und Natur [2006], Frankfurt ${ }^{3} 2015,163$.

${ }^{23}$ Das logische Verhältnis der verschiedenen Formulierungen des kategorischen Imperativs ist allerdings in der Fachliteratur umstritten.
} 
Auch bei Ernst Tugendhat finden wir dieses Schwanken zwischen Apriori und Empirie. Zu Recht bemerkt er, dass bei Habermas in der idealen Sprechsituation das vorausgesetzt wird, was allererst begründet werden sollte. Im Gegensatz dazu lässt Tugendhat keinerlei Apriori gelten. Der Mensch sei nicht von sich aus auf Moralität hin ausgerichtet, sondern er müsse sich dazu entschliessen, denn unser Bewusstsein habe keine »transzendente Dimension «24.

Aber dann arbeitet er doch, wie Habermas, mit metaphysischen Ideen. Die Moral habe es mit »irgendwie absoluten Verpflichtungen zu tun." Der unparteiische Betrachter, der zu diesem Zweck unterstellt werden müsse, sei eine "regulative Idee« und schliesslich stützt er sich auf die "inclinationes naturales" wie bei Thomas von Aquin: Wir setzen z.B. voraus, dass alle Menschen nicht geschädigt werden wollen usw. ${ }^{25}$ Obwohl er das Wort für seine eigene Position vermeiden möchte, ist er sich im Grunde im Klaren, dass es ohne Naturrecht nicht geht, vor allem in Bezug auf die Menschenrechte: "Es ist wegen ihrer Zweideutigkeit besser, die Rede von Naturrechten zu vermeiden; ihr guter Sinn besteht einfach darin, dass es sich hier um Rechte handelt, die, wenn wir sie überhaupt anerkennen, vor aller positiven Rechtssetzung gelten. $\aleph^{26}$ Also doch!

Besonders radikal ist Norbert Hoerster. In seinem Buch über Moralbegründung "widerlegt« er alle solchen Begründungen, besonders aber die aus dem Naturrecht. Dabei versteht er unter "Naturrecht" eine Position, die sich auf die äussere Natur bezieht, also auf die Dinge der Natur, statt auf die Natur der Dinge. Wir haben schon gesehen, dass das nicht der Sinn des Naturrechts sein kann, weil es sonst nichts sein würde als ein System grober naturalistischer Fehlschlüsse.

Über seine eigene Position sagt Hoerster: »Die Akzeptanz einer Norm kann nach der hier vertretenen Sichtweise nur dann als rational betrachtet werden, wenn sie auch im Interesse dessen liegt, der die Norm akzeptiert", denn für den Durchschnittsmenschen überwiegen die Vorteile des Moralischen seine Nachteile, denn "nicht jeder hat das Zeug zum Mafioso. "Ausserdem seien wir an Fairness interessiert: "Ich will ein faires Leben und nicht das Leben eines parasitären Trittbrettfahrers führen. $\otimes^{27}$

Das ist nun aber etwas verworren. Ich bin nur deshalb kein Mafioso, weil es zu anstrengend ist? Das sehen die Mafiosi anders, für die das Interesse am grossen Geld das Interesse an einem anstren-

${ }^{24}$ Ernst Tugendhat,Vorlesungen über Ethik, Frankfurt 1993, 15, 165.

${ }^{25}$ Ebd., 40, 313, 316.

${ }^{26}$ Ebd., 346.

${ }^{27}$ Norbert Hoerster, Wie lässt sich Moral begründen?, München 2014, 119, 124-126. 
genden, aber guten, Leben überwiegt und ganz allgemein gilt doch das Leben der Moral als anstrengender als das Leben eines Egoisten. Aber wenn schon: Würden wir ein Leben, das es sich möglichst bequem macht, "moralisch" nennen? Und wenn wir am Leben unter fairen Bedingungen interessiert sind, kommen wir dann nicht in die Nähe der "inclinationes naturales" des Thomas von Aquin? In diese Richtung deutet auch die folgende Aussage: »Eine Moralnorm ist genau dann objektiv begründet, wenn ihre Geltung im Interesse aller Normadressaten liegt." Die menschliche Natur sei so beschaffen, dass die wichtigsten Interessen aller Menschen gleichgerichtet sind. ${ }^{28}$ Dieser Eindruck, dass Norbert Hoersters Moralkonzept eben doch eine Naturrechtslehre zugrundeliegt, verstärkt sich bei der Lektüre seines Buches »Ethik und Interesse«.

In diesem Buch ist sein Rückgriff auf das Naturrecht womöglich noch deutlicher. Hier nennt er weitere Interessen: Überleben, körperliche Unversehrtheit, Lügenverbot, Bewegungs- und Handlungsfreiheit, das Interesse, nicht angelogen zu werden usw. Da manche diesem Interessenkalkül nicht zustimmen werden, führt Hoerster das "aufgeklärte Interesse« ein. Er lehne zwar das Naturrecht ab, es gebe aber "natürliche Interessen", die allen Menschen gemeinsam seien. Er spricht sogar ganz essentialistisch von einer "menschlichen Natur ${ }^{29}$. Aber was sind das anderes als naturrechtliche Bestimmungen und woher nehmen wir die Kriterien für die Differenz zwischen beliebigen und aufgeklärten Interessen?

Das Fazit dieser Überlegungen mag vielleicht etwas mager erscheinen: Moralität kommt um einen minimalen Rekurs auf das Naturrecht nicht aus. Das liegt schon allein, wie sich gezeigt hat, darin, dass sie das Verallgemeinerungsprinzip enthalten muss. Wir würden keinen Menschen für moralisch halten, der sich vom Verbot der Lüge ausnimmt oder der damit einverstanden wäre, dass Putin oder Trump lügen dürfen, wir aber nicht und wir werden es nicht akzeptieren, dass bei uns die Menschenrechte gelten, in China aber

\footnotetext{
${ }^{28}$ Ebd, 132, 135.

${ }^{29}$ Ders., Ethik und Interesse, Stuttgart 2003, 168, 172f, 176. Otfried Höffe spricht konsequenter von "transzendentalen Interessen« (Nach Pollmann/ Menke, Philosophie der Menschenrechte, 50).
} 
nicht. Das impliziert aber eine Minimalmetaphysik, ohne die es keine Ethik und keine Menschenrechte geben kann.

- Prof. Dr. Hans-Dieter Mutschler war von 2003 bis 2018 Inhaber des Lehrstuhls für Naturphilosophie an der philosophisch-pädagogischen Hochschule Ignatianum in Krakau. Er ist Herausgeber von Karl Rahners Schriften zur Naturwissenschaft und zum Marxismus. Des Weiteren lehrt er an der Universität Zürich und an der PhilosophischTheologischen Hochschule Sankt Georgen in Frankfurt am Main. Seine philosophischen Arbeiten beziehen sich auf Fragen der Naturphilosophie und auf das Verhältnis von Naturwissenschaft und Theologie. 